\title{
Mortality among patients with pleural effusion undergoing thoracentesis
}

\author{
Erin M. DeBiasi ${ }^{1}$, Margaret A. Pisani ${ }^{1}$, Terrence E. Murphy ${ }^{2}$, Katy Araujo², \\ Anna Kookoolis ${ }^{1}$, A. Christine Argento ${ }^{3}$ and Jonathan Puchalski ${ }^{1}$
}

Affiliations: ${ }^{1}$ Division of Pulmonary, Critical Care and Sleep Medicine, Yale University, New Haven, CT, USA. ${ }^{2}$ Claude D. Pepper Older Americans Independence Center at Yale, Program on Aging. New Haven, CT, USA. ${ }^{3}$ Division of Pulmonary and Critical Care Medicine, Emory University, Atlanta, GA, USA.

Correspondence: Jonathan Puchalski, Division of Pulmonary, Critical Care and Sleep Medicine, Yale University, 15 York Street, LCl 100, New Haven, CT 06510, USA. E-mail: Jonathan.puchalskidayale.edu

ABSTRACT Of the 1.5 million people diagnosed with pleural effusion in the USA annually, $\sim 178000$ undergo thoracentesis. While it is known that malignant pleural effusion portends a poor prognosis, mortality of patients with nonmalignant effusions has not been well studied.

This prospective cohort study evaluated 308 patients undergoing thoracentesis. Chart review was performed to obtain baseline characteristics. The aetiology of the effusions was determined using standardised criteria. Mortality was determined at 30 days and 1 year.

247 unilateral and 61 bilateral thoracenteses were performed. Malignant effusion had the highest 30-day (37\%) and 1-year (77\%) mortality. There was substantial patient 30-day and 1-year mortality with effusions due to multiple benign aetiologies (29\% and 55\%), congestive heart failure (22\% and 53\%), and renal failure (14\% and 57\%, respectively). Patients with bilateral, relative to unilateral, pleural effusion were associated with higher risk of death at 30 days and 1 year (17\% versus $47 \%$ (hazard ratio (HR) $2.58,95 \%$ CI $1.44-4.63$ ) and $36 \%$ versus $69 \%$ (HR 2.32 , 95\% CI 1.55-3.48), respectively).

Patients undergoing thoracentesis for pleural effusion have high short- and long-term mortality. Patients with malignant effusion had the highest mortality followed by multiple benign aetiologies, congestive heart failure and renal failure. Bilateral pleural effusion is distinctly associated with high mortality.

@ERSpublications

This study shows malignant, bilateral and effusion due to multiple benign aetiologies has significant mortality http://ow.ly/JJ4hC

Received: Nov 242014 | Accepted after revision: Feb 242015 | First published online: April 022015

Support statement: This study was funded by the Yale Claude D. Pepper Older Americans Independence Center (grant P30AG21342).

Conflict of interest: None declared.

Copyright @ERS 2015 


\section{Introduction}

Pleural effusions are diagnosed annually in $>1.5$ million people in the USA [1] and are caused by various medical conditions. The most common causes of pleural effusion are, in descending order, congestive heart failure (CHF), pleural infection and malignancy [2]. CHF alone is responsible for about one-third of all cases in the USA each year [1]. Although malignant pleural effusions are associated with high mortality, there is little in the literature regarding mortality of patients with nonmalignant pleural effusion.

Bilateral pleural effusions are common, accounting for $15 \%$ of the cases in non-critically ill patients, and up to $55 \%$ of those in the intensive care population. The aetiology of bilateral pleural effusion includes CHF, coronary artery bypass graft, fluid overload, liver and renal failure, and malignancy $[3,4]$. To our knowledge, the association between bilateral pleural effusion and mortality has not been previously reported.

In this study, we determined 30-day and 1-year mortality for patients with pleural effusions of differing aetiologies. We also compared the mortality rates between patients with unilateral and bilateral pleural effusion.

\section{Methods}

This prospective observational cohort study was performed at Yale-New Haven Hospital (YNHH), New Haven, CT, USA, a tertiary care academic hospital with $>1000$ beds, and approved by the Institutional Review Board (IRB) of YNHH. All patients who underwent thoracentesis between December 2010 and December 2011 by the interventional pulmonary service were approached for enrolment. In addition to informed consent for the procedure, IRB-approved informed consent for the study was obtained from each participant. The interventional pulmonary service at $\mathrm{YNHH}$ performs the majority of the thoracenteses on both in- and outpatients, with a minority performed by interventional radiology, surgical services and others. Chart review and patient interviews were performed to identify baseline patient characteristics and demographics, including medical comorbidities.

All thoracenteses were performed using ultrasound guidance (S-ICU; SonoSite, Bothell, WA, USA) in a sterile fashion using the Safe-T-Centesis kit (Cardinal Health, Dublin, OH, USA). Fluid was aspirated manually and routinely sent for analysis including cell count with differential count, total protein, lactate dehydrogenase, glucose, $\mathrm{pH}$, cultures (bacterial, fungal and acid-fast bacilli) and cytology. Flow cytometry was requested if lymphoma was a reasonable concern. Light's criteria were used to classify each effusion as transudate or exudate [5]. When bilateral effusions were present, concurrent bilateral thoracenteses were always performed [3].

Table 1 provides the definitions used to categorise the aetiology of the pleural effusion. Patients were classified as having a certain aetiology if they met one of the characteristics listed in a category. Mortality was determined at 30 days and 1 year using chart review.

\section{Statistical analysis}

The demographics and occurrence of common comorbidities were described. Unadjusted 30-day and 1-year mortalities were calculated and diagrammed for subgroups according to the following hierarchy: laterality (bilateral versus unilateral) and malignancy (yes versus no). Nonmalignant effusion was further classified by type of effusion (exudate versus transudate and mismatch, or dissimilar between sides, for some bilateral). Multivariable proportional hazards Cox models evaluated associations between short-term (30-day) and long-term (1-year) mortality, and the following explanatory variables. 1) Age in years. 2) Male sex (referent to female). 3) Nonwhite race. 4) Number of comorbidities. 5) Bilateral thoracentesis (referent to unilateral). 6) Indicators for each of the following aetiologies (referent to malignancy): CHF, infectious disease, liver failure, renal failure, multiple benign aetiologies and paramalignancy. KaplanMeier plots of adjusted survival probabilities were created for 30-day and 1-year mortality, and stratified for bilateral versus unilateral thoracentesis. All analyses were performed using SAS v9.3 (SAS Institute, Cary, NC, USA), and $\mathrm{p}<0.05$ (two-tailed) was used to denote statistical significance.

\section{Results}

A total of 320 patients over the study period were enrolled and 308 patients were included in the analysis. 12 participants were excluded for multiple reasons including incomplete clinical data or insufficient pleural fluid for thoracentesis after enrolment in the study. Baseline patient characteristics are presented in table 2. The majority of thoracenteses were performed on inpatients and patients with a history of cancer or a cardiac condition (53\% and $80 \%$, respectively). A total of 247 unilateral and 61 bilateral thoracenteses were performed. Overall mortality across all groups was 21\% (65 out of 308 patients) at 30 days and $51 \%$ (158 out of of 308 patients) at 1 year, with no procedure-related deaths. 
TABLE 1 Categories of pleural effusion aetiologies and their corresponding definitions

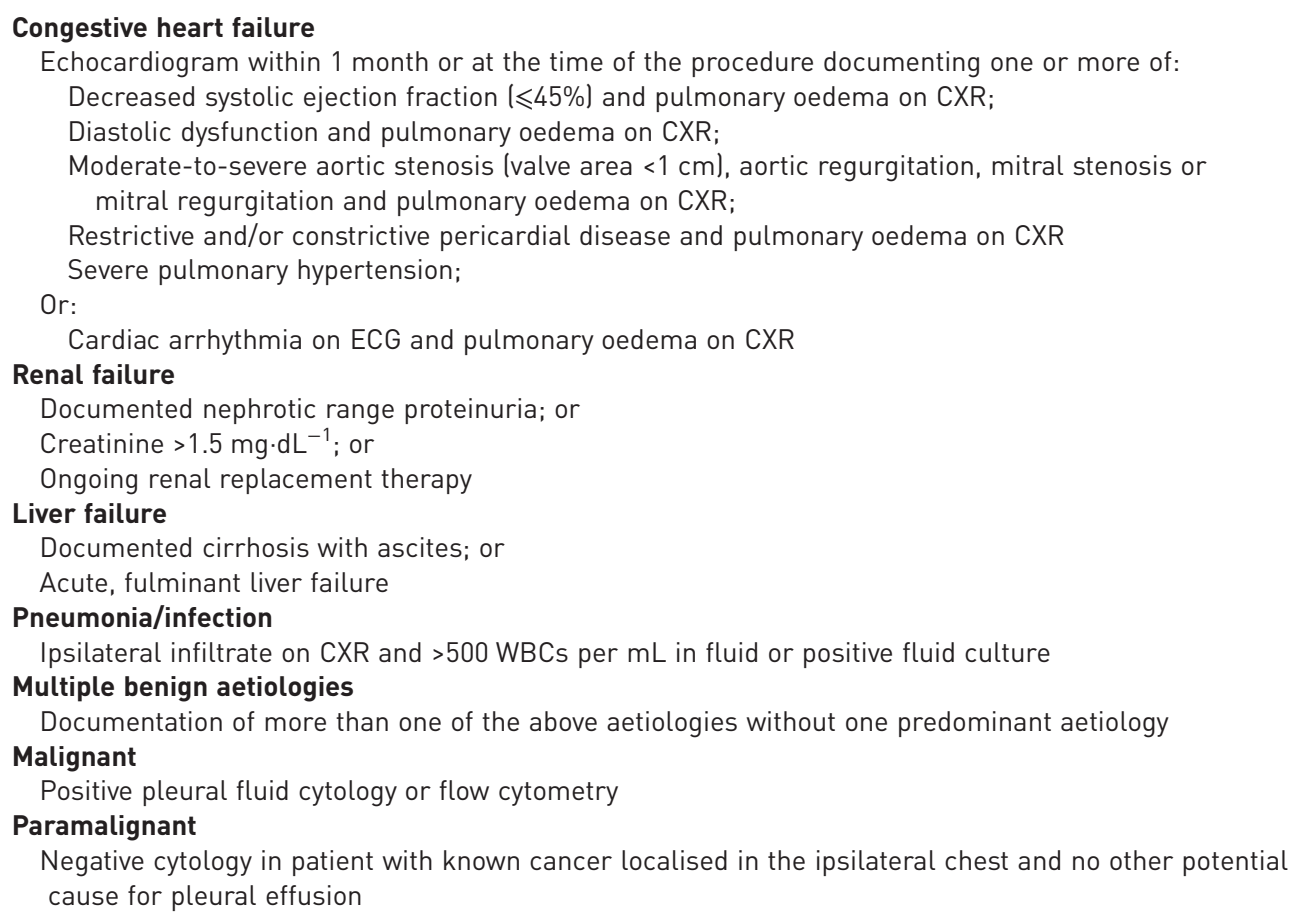

Liver failure

Documented cirrhosis with ascites; or

Acute, fulminant liver failure

Pneumonia/infection

Ipsilateral infiltrate on CXR and $>500$ WBCs per $\mathrm{mL}$ in fluid or positive fluid culture

Multiple benign aetiologies

Documentation of more than one of the above aetiologies without one predominant aetiology

Malignant

Positive pleural fluid cytology or flow cytometry

Paramalignant

Negative cytology in patient with known cancer localised in the ipsilateral chest and no other potential cause for pleural effusion

CXR: chest radiography; WBC: white blood cell.

Figure 1 depicts the pleural effusion characteristics and corresponding unadjusted mortality at 30 days and 1 year. Malignant pleural effusion was identified in $27 \%$ of patients. The percentage of patients with malignancy as their aetiology was similar in patients with unilateral and bilateral effusion. The pleural fluid type of nonmalignant effusion differed by whether it was bilateral; most unilateral nonmalignant

\section{TABLE 2 Baseline characteristics and demographics ( $n=308$ )}

Age years
Male
Nonwhite race
Inpatient
Number of comorbidities
Arthritis
Diabetes
GI conditions ${ }^{\#}$
Mental issues
Liver failure/cirrhosis
Renal failure
Obesity
Autoimmune disease
Cancer
Neurological disease
Pulmonary disease
Sleep apnoea
Heart condition

Data are presented as mean $\pm S D$ or $n(\%)$. Missing data: race $(n=10)$; arthritis $(n=2)$; gastrointestinal (GI) conditions ( $n=2)$; obesity $(n=2)$; autoimmune disease $(n=2)$. \# : digestive problems (such as gastro-oesophageal reflux disease, Crohn's disease, ulcerative colitis or irritable bowel syndrome); ": anxiety, depression or memory problems; ${ }^{+}$: stroke, ministroke or seizure; ${ }^{\S}$ : asthma, chronic obstructive pulmonary disease or interstitial lung disease; ${ }^{f}$ : myocardial infarction, angina, coronary disease, arrhythmia, hypertension, hyperlipidaemia, congestive heart failure or a pacemaker. 


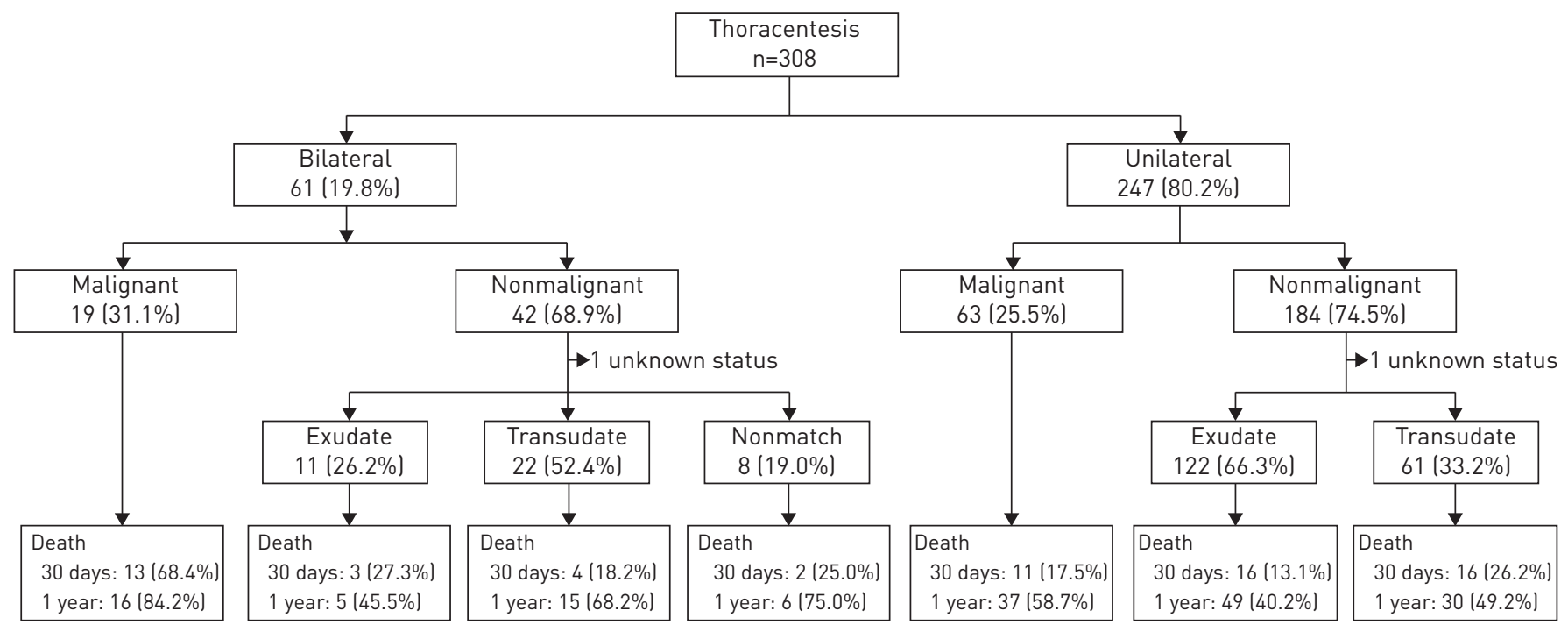

FIGURE 1 Pleural effusion characteristics and corresponding unadjusted mortality at 30 days and 1 year.

cases were exudates whereas most bilateral nonmalignant cases were transudates. As calculated from figure 1, 30-day and 1-year mortality of patients with unilateral effusion was $17 \%$ and $47 \%$, compared with $36 \%$ and $69 \%$ in patients with bilateral effusion. Relative to patients with unilateral effusion, unadjusted mortality of patients with bilateral effusion was higher at 30 -days $(\mathrm{p}=0.0003)$ and at 1 -year $(\mathrm{p}=0.0001)$.

Figure 2 depicts mortality based on the aetiology of the pleural effusion at 30 days and 1 year. Patients with malignant and paramalignant pleural effusion had the highest 30 -day (37\% and $28 \%$, respectively) and 1-year mortality (77\% and $75 \%$, respectively). Patients whose effusions were attributed to multiple benign aetiologies also had high mortality at 30 days and 1 year (29\% and 55\%, respectively), as did patients with pleural effusion singularly due to CHF (22\% and 53\%, respectively). Patients with infection, including parapneumonic effusion and empyema, also exhibited substantive 30-day and 1-year mortality (11\% and 26\%, respectively).

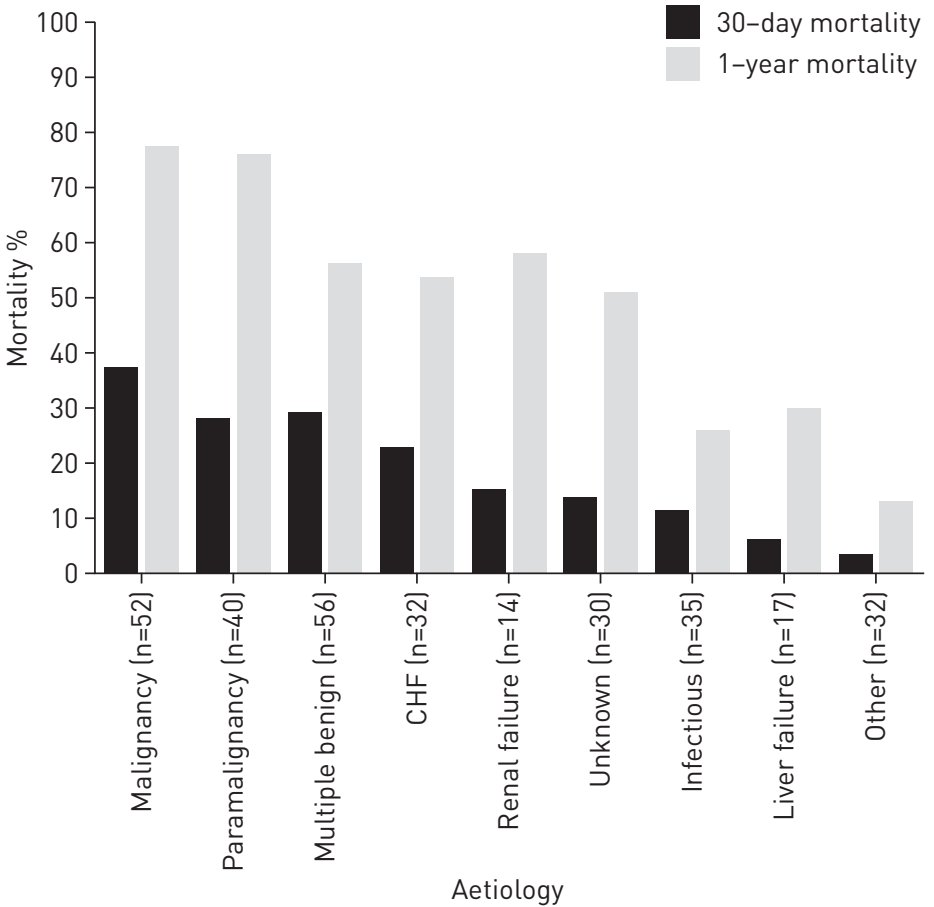

FIGURE 2 Percent mortality based on the aetiology of the pleural effusion at 30 days and 1 year. The number of pleural effusions with the corresponding aetiology is shown. CHF: congestive heart failure. 

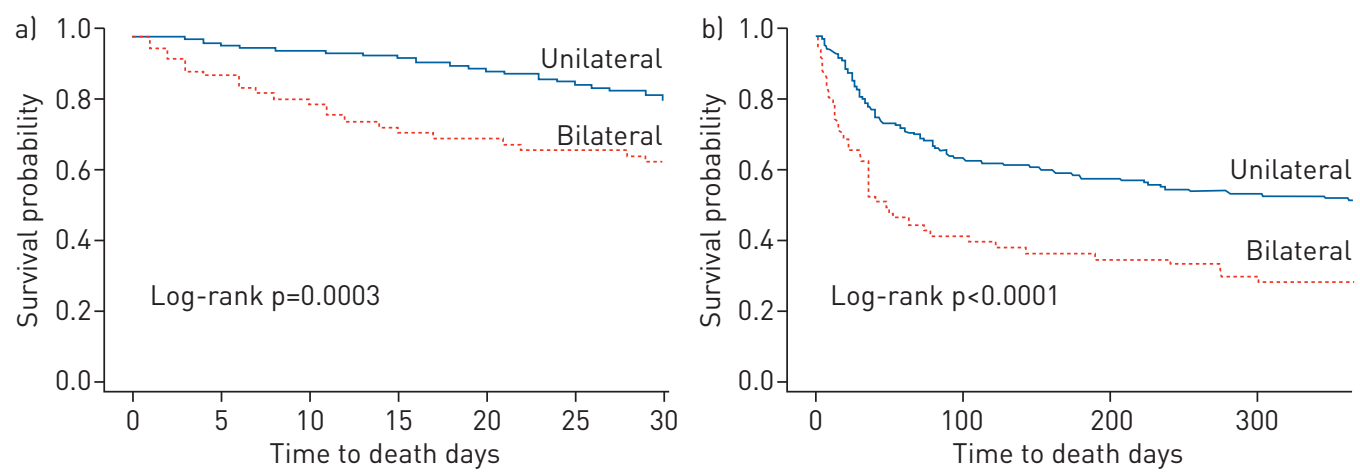

FIGURE 3 Kaplan-Meier survival plots for a) short- and b) long-term mortality (bilateral versus unilateral pleural effusion).

Figure 3 contrasts the Kaplan-Meier survival curves of patients with unilateral and bilateral pleural effusion at 30 days and 1 year. In both time periods, the presence of bilateral pleural effusion was associated with significantly lower survival probability.

Table 3 presents associations between the following explanatory variables and short term (30-day) and long-term (1-year) mortality: age, sex, race, number of comorbidities, aetiology (relative to malignancy) and bilateral (relative to unilateral). Number of comorbidities was the only covariate significantly associated with mortality at both 30-days and 1-year (hazard ratio (HR) 1.21 (95\% CI 1.05-1.39, p=0.009) and HR 1.19 (95\% CI 1.09-1.30, p=0.0002), respectively). Even after adjustment for these covariates, the presence of bilateral effusion, relative to unilateral effusion, was significantly associated with higher 30-day and 1-year mortality (HR 2.58 (95\% CI 1.44-4.63, p=0.002) and HR 2.32 (95\% CI 1.55-3.48, p<0.001), respectively).

\section{Discussion}

Encountered frequently by chest physicians and other medical subspecialists, pleural effusion is a significant cause of dyspnoea and patient suffering. Therapeutic thoracentesis may provide symptomatic relief, and care directed at the underlying aetiology often determines whether the effusion recurs. This study is the first to evaluate both short- and long-term mortality in patients who have undergone thoracentesis, and whose pleural effusion is attributed to a variety of aetiologies.

The short-term mortality in patients undergoing thoracentesis for pleural effusion is high, with $>20 \%$ of patients dying within 30 days. In the USA, the Centers for Medicare and Medicaid Services and the Hospital Quality Alliance began publically reporting 30-day mortality measures for acute myocardial

TABLE 3 Adjusted associations between characteristics of thoracentesis/pleural effusion and mortality among patients undergoing thoracentesis

\begin{tabular}{|c|c|c|c|c|}
\hline \multirow[t]{2}{*}{ Characteristic } & \multicolumn{2}{|c|}{ 30-day mortality } & \multicolumn{2}{|c|}{ 1-year mortality } \\
\hline & Hazard ratio $(95 \%$ CI $)$ & p-value & Hazard ratio $(95 \% \mathrm{CI})$ & p-value \\
\hline \multicolumn{5}{|l|}{ Thoracentesis ${ }^{\#}$} \\
\hline Bilateral & $2.58(1.44-4.63)$ & 0.002 & $2.32(1.55-3.48)$ & $<0.001$ \\
\hline \multicolumn{5}{|l|}{ Aetiology" } \\
\hline $\mathrm{CHF}$ & $0.33(0.13-0.85)$ & 0.021 & $0.28(0.15-0.54)$ & 0.001 \\
\hline Infectious disease & $0.20(0.09-0.45)$ & $<0.001$ & $0.20(0.12-0.33)$ & $<0.001$ \\
\hline Liver disease & $0.09(0.01-0.71)$ & 0.022 & $0.17(0.07-0.45)$ & 0.003 \\
\hline Multiple benign aetiologies & $0.43(0.20-0.90)$ & 0.024 & $0.36(0.21-0.61)$ & $<0.001$ \\
\hline Paramalignant & $0.59(0.28-1.25)$ & 0.164 & $0.87(0.54-1.40)$ & 0.568 \\
\hline Renal disease & $0.24(0.05-1.07)$ & 0.061 & $0.37(0.17-0.81)$ & 0.013 \\
\hline \multicolumn{5}{|l|}{ Covariates } \\
\hline Age in years & $0.99(0.97-1.01)$ & 0.430 & $1.01(0.97-1.02)$ & 0.361 \\
\hline Male sex & $0.96(0.58-1.60)$ & 0.877 & $1.07(0.77-1.40)$ & 0.686 \\
\hline Nonwhite race & $0.63(0.28-1.42)$ & 0.262 & $0.98(0.61-1.58)$ & 0.937 \\
\hline Number of comorbidities & $1.21(1.05-1.39)$ & 0.009 & $1.19(1.09-1.30)$ & $<0.001$ \\
\hline
\end{tabular}


infarction and CHF in 2007, and for pneumonia in 2008. Hospitals may be penalised for high readmission and mortality rates, and rewarded for significant improvements in such measures. It stands to reason that patients with cardiac disease and pneumonia develop pleural effusion, and that a heightened awareness of pleural effusion's role in these diseases may influence treatment decisions. Recognising that pleural effusion is associated with high mortality may prompt more aggressive treatment of the disease process driving its manifestation.

\section{Unilateral versus bilateral effusion}

The study is novel in evaluating mortality associated with bilateral effusions. We demonstrated a significant positive association with mortality at both 30 days and 1 year in patients with bilateral versus unilateral pleural effusion. Because each group had similar percentages of malignant effusions, that single aetiology does not fully account for the difference. A large study of patients with community-acquired pneumonia demonstrated that patients with bilateral pleural effusion had significantly higher 30-day mortality than those with unilateral (26\% versus $5.9 \%$, respectively) [6]. We believe this is not attributable to the bilateral nature of the intervention, but rather, an indication of seriously advanced disease.

\section{Pleural effusion in malignancy}

It is well known that dissemination of malignant cells into the pleural space is a marker of advanced malignancy. Survival of patients with malignant pleural effusion differs by the type of primary tumour, with the shortest survival being associated with lung cancer [7]. Numerous studies, including this one, demonstrate that malignant pleural effusion is associated with poor outcomes [8-11]. In this study, malignant and paramalignant effusion had similar mortality outcomes at both 30 days and 1 year, justifying our classification of paramalignant as those that are cytologically negative but most likely secondary to cancer. In our study, the highest mortality associated with pleural effusion was observed in patients with underlying malignancy. One-third of these patients died within 30 days of thoracentesis and two-thirds within 1 year. Patients with bilateral malignant pleural effusion are unique and have high 30 day mortality. These patients, in particular, may be better suited to symptom management via therapeutic thoracentesis than more invasive procedures, such as pleurodesis [7]. For these patients, palliation and consideration of hospice care may be more appropriate.

\section{Pleural effusion in CHF}

In the USA, annual overall mortality for patients with CHF ranges from $10 \%$ to $16 \%[12,13]$. The patients in this study with pleural effusion due to CHF had short-term and annual mortality that was nearly triple that reported in other studies. Our results align with recent data reporting annual mortality of $33 \%$ in patients hospitalised with acute decompensation of chronic heart failure [14]. Data were recently published indicating that pleural effusion in acute decompensated CHF does not correlate with increased mortality at 6 months [15]. Notably, in that study, the majority of the pleural effusion cases were mild, identified only by chest radiography, and did not necessitate thoracentesis. We believe that pleural effusion due to $\mathrm{CHF}$ should be viewed as further evidence of acute decompensation and, in order to prevent readmission and death, advocate more aggressive therapy.

\section{Infectious pleural effusion}

Pleural effusion in the setting of pulmonary infection is common, occurring in up to $9 \%$ of patients with pneumonia, and is a poor prognostic sign, particularly if the effusion is moderate to large in size, bilateral and/or associated with empyema $[6,16-18]$. In our study, patients with infection contributing to their effusion had similar mortality as prior studies, with 30 -day and 1-year mortality of $11 \%$ and $26 \%$, respectively. The treatment of complicated pleural space infection with tissue plasminogen activator and DNase improves outcome [19]. Appropriate management of these cases includes early identification of empyema via thoracentesis, treatment with appropriate antibiotics, chest tube drainage and surgery when indicated.

\section{Pleural effusion in renal failure}

A small number of patients in this study had pleural effusion due to renal failure, which is most often due to hypervolaemia but can also be secondary to exudative uraemic pleuritis [20]. Sometimes they also occur via communication between the peritoneum and thorax through the diaphragm in peritoneal dialysis patients [21]. Mortality associated with effusion due to kidney disease has not been well studied. A small study of patients undergoing peritoneal dialysis demonstrated that patients with exudative pleural effusion, probably from uraemic pleuritis, had high mortality (60\% at 3 years) [22]. In our small cohort of 14 patients with renal failure as the single cause of their effusion, their 30 -day and 1-year mortality was $14 \%$ and $57 \%$, respectively. 
Pleural effusion associated with multiple benign aetiologies

No other study has characterised patients with pleural effusion due to multiple benign aetiologies and their mortality. Their high mortality is probably multifactorial. Common benign conditions contributing to development of pleural effusion include cardiorenal and hepatorenal disease. Underlying malnutrition and hypoalbuminaemia associated with these multiorgan disease states probably contribute to increased mortality. Pleural effusion in these patient populations may be a marker of their illness severity. In the setting of multiple benign aetiologies, these results provide evidence that multiorgan dysfunction is worse than single-organ failure.

\section{Study strengths and limitations}

Although this study is a well-characterised cohort of medical patients undergoing thoracentesis, it has general applicability to the broader inpatient medical population. The patient sample size is, to our knowledge, the largest published to date that evaluates mortality by aetiology of pleural effusion. It is also the first study to report the association between bilateral pleural effusion and mortality.

Limitations of this study include that it was performed at a single academic institution and the majority of patients had diseases with effusion secondary to their medical aetiology. Because the majority of thoracenteses in our hospital are performed by dedicated proceduralists, patients who had their procedures performed by other providers were not enrolled. Because this cohort is limited to patients who underwent thoracentesis, we cannot say anything about mortality in patients with pleural effusion who did not undergo thoracentesis. A recent paper attempted to address this question retrospectively by identifying all patients who had pleural effusion per chest imaging at time of hospital admission [23]. That study determined 30-day and 1-year mortality of patients who had thoracentesis performed versus those who did not. 30-day mortality trended lower in the thoracentesis subgroup (9\% versus $16 \%$ ) and trended higher at 1 year (36\% versus $31 \%$ ). However, neither of these trends was statistically significant as this was a small, underpowered retrospective study. Mortality rates were, on a whole, lower in this study compared to the current study, also probably a reflection of the small sample size of 11 patients who underwent thoracentesis over a 1-month period.

Pleural effusion serves as a marker of disease and is influenced by many factors. In CHF, for example, multiple variables are involved that subsequently influence its course, including medication regimens, patient compliance, ventricular assist devices, copresence of coronary artery disease and ischaemia, and arrhythmia. Similar variability is encountered in patients with renal failure, infection and malignancy. Importantly, this study does not evaluate the influence on mortality of different treatment regimens to control effusion. In this sense, the pleural disease serves more as a marker of severe disease, but does not guide a specific way to decrease mortality. Rather, given the degree of mortality seen in patients with pleural effusions, the requirement of undergoing a thoracentesis for diagnostic or therapeutic purposes should indicate that the patient's underlying disease process is advanced and that maximal therapy aimed at treating the underlying aetiology is paramount.

\section{Conclusions}

Patients who undergo thoracentesis as a treatment for pleural effusion are encountered across all aspects of medicine. In addition to malignant effusion, paramalignant effusion, effusion due to multiple benign aetiologies and bilateral effusion all have considerable 30-day and 1-year mortality. Malignant effusion had the highest 30-day (37\%) and 1-year (77\%) mortality, followed by effusions due to multiple benign aetiologies (29\% and 55\%), CHF (22\% and 53\%) and renal failure (14\% and 57\%). Patients with bilateral pleural effusions had a significantly higher risk of death than patients with unilateral effusions at both time-points. Caregivers encountering patients with pleural effusion should be aware of their heightened mortality risks and aggressive management of the underlying aetiology is warranted.

\section{References}

$1 \quad$ Light RW. Pleural Diseases. Philadelphia, Lippincott Williams \& Wilkins, 2007.

Light RW. Pleural effusions. Med Clin North Am 2011; 95: 1055-1070.

Puchalski JT, Argento AC, Murphy TE, et al. Etiologies of bilateral pleural effusions. Respir Med 2013; 107: 284-291.

4 Kalomenidis I, Rodriguez M, Barnette R, et al. Patient with bilateral pleural effusion: are the findings the same in each fluid? Chest 2003; 124: 167-176.

5 Light RW, Macgregor MI, Luchsinger PC, et al. Pleural effusions: the diagnostic separation of transudates and exudates. Ann Intern Med 1972; 77: 507-513.

6 Hasley PB, Albaum MN, Li YH, et al. Do pulmonary radiographic findings at presentation predict mortality in patients with community-acquired pneumonia? Arch Intern Med 1996; 156: 2206-2212.

7 Roberts ME, Neville E, Berrisford RG, et al. Management of a malignant pleural effusion: British Thoracic Society Pleural Disease Guideline 2010. Thorax 2010; 65: Suppl. 2, ii32-ii40. 
Pilling JE, Dusmet ME, Ladas G, et al. Prognostic factors for survival after surgical palliation of malignant pleural effusion. J Thorac Oncol 2010; 5: 1544-1550.

9 Ozyurtkan MO, Balci AE, Cakmak M. Predictors of mortality within three months in the patients with malignant pleural effusion. Eur J Intern Med 2010; 21: 30-34

10 Heffner JE, Nietert PJ, Barbieri C. Pleural fluid $\mathrm{pH}$ as a predictor of survival for patients with malignant pleural effusions. Chest 2000; 117: 79-86.

11 Tremblay A, Michaud G. Single-center experience with 250 tunnelled pleural catheter insertions for malignant pleural effusion. Chest 2006; 129: 362-368.

12 Packer M, Coats AJS, Fowler MB, et al. Effect of carvedilol on survival in severe chronic heart failure. $N$ Engl $J$ Med 2001; 344: 1651-1658.

13 Cleland JG, Daubert J, Erdmann E, et al. The effect of cardiac resynchronization on morbidity and mortality in heart failure. N Engl J Med 2005; 352: 1539-1549.

14 Lassus JP, Siirila-Waris K, Nieminen MS, et al. Long-term survival after hospitalization for acute heart failure differences in prognosis of acutely decompensated chronic and new-onset acute heart failure. Int J Cardiol 2013; 168: 458-462.

15 Davutoglu V, Yildirim C, Kucukaslan H, et al. Prognostic value of pleural effusion, CA-125 and NT-proBNP in patients with acute decompensated heart failure. Kardiol Pol 2010; 68: 771-778.

16 Sahn SA. Diagnosis and management of parapneumonic effusions and empyema. Clin Infect Dis 2007; 45: 1480-1486.

17 Ferguson AD, Prescott RJ, Selkon JB, et al. The clinical course and management of thoracic empyema. QJM 1996; 89: 285-289.

18 Davies CW, Kearney SE, Gleeson FV, et al. Predictors of outcome and long-term survival in patients with pleural infection. Am J Respir Crit Care Med 1999; 160: 1682-1687.

19 Rahman NM, Maskell NA, West A, et al. Intrapleural use of tissue plasminogen activator and DNase in pleural infection. N Engl J Med 2011; 365: 518-526.

20 Bakirci T, Sasak G, Ozturk S, et al. Pleural effusion in long-term hemodialysis patients. Transplant Proc 2007; 39: 889-891.

21 Lew SQ. Hydrothorax: pleural effusion associated with peritoneal dialysis. Perit Dial Int 2010; 30: 13-18.

22 Kwan BC, Chow KM, Pang WF, et al. Unexplained exudative pleural effusion in chronic peritoneal dialysis patients. Perit Dial Int 2010; 30: 534-540.

23 Kookoolis AS, Puchalski JT, Murphy TE, et al. Mortality of hospitalized patients with pleural effusions. J Pulm Respir Med 2014; 4: 184. 